\title{
OpenEarth: using Google Earth as outreach for NCK's data
}

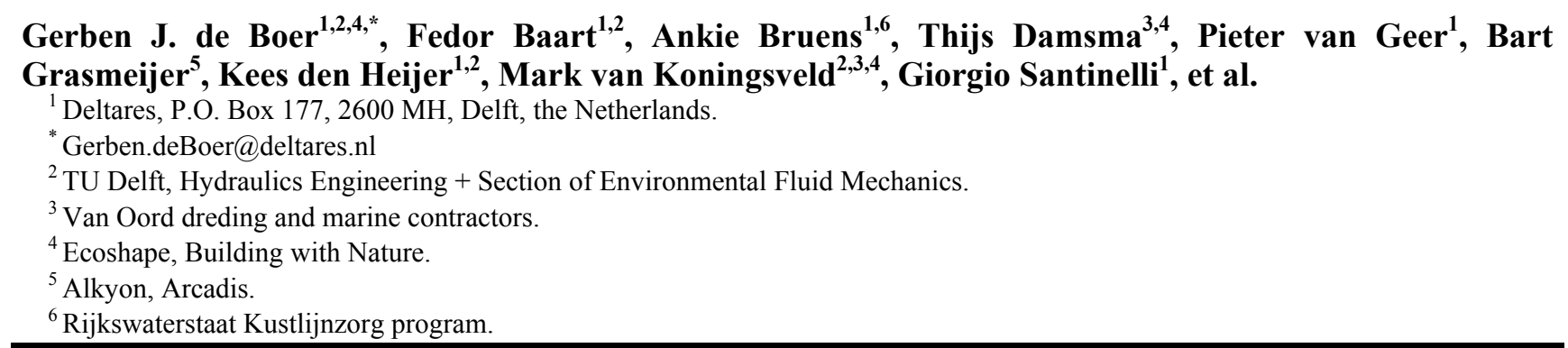

\begin{abstract}
In 2003 various projects at Deltares and the TU-Delft merged their toolboxes for marine and coastal science and engineering into one toolbox, culminating in 2008 in an open source release, known as OpenEarthTools (OET). OpenEarth adopts the wikipedia approach to growth: web 2.0 crowd sourcing. All users are given full write access to help improve the collection. Quality is assured by version control, tracking all changes. OpenEarth started as a social experiment to investigate whether crowd sourcing was possible in our community of marine and coastal researchers. The answer is yes: over 1000 users registered, now enjoying over 5000 contributions from over 100 contributors. The most important asset is a general toolbox to plot any data type in Google Earth. With this toolbox it has become very easy for marine and coastal experts to disseminate their data via Google Earth. It enables the NCK community to make its data available to end-users and the general public with only little effort. They can now consume our data as simple as watching YouTube: DataTube. In this paper it is shown that OpenEarth has added important value by analyzing a wide range of marine and coastal data types from NCK simultaneously in Google Earth. To match the traditional gap between specialist knowledge and end users, Google Earth is shown to be a very powerful tool. The possibilities for outreach by NCK are manifold.
\end{abstract}

\section{INTRODUCTION}

The Netherlands is one of the flattest, lowest and flood-prone countries around the world. Yet, the Netherlands has probably the best digital elevation model (DEM) of the world: $1 \mathrm{~m}$ nation-wide coverage with the AHN DEM [AHN]. For the dynamic dune rows and foreshore a time dependent DEM is available between 1996 and present at $5 \mathrm{~m}$ resolution. This data is made openly available by Rijkswaterstaat, and allows for detailed study of marine and coastal dynamics, not only by analyzing trends in the wealth of data itself, but also as input or validation for a wide range of models. The cost of such mass gathering of data, Lidar in this case, will drop in the future, leading to an even bigger wealth of data. This trend is known as the Digital Data Deluge [e.g., e-IRG, 2010]. However, the scientific use of this abundance of data is still far from optimal. The main reason for this is that the dedicated, often self-made software used by of marine and coastal scientists and engineers cannot handle such large quantities of data and can hardly handle the wide variety. This applies even more to the use of this wealth of data by policy makers and the general public. These latter groups cannot use the dedicated software used by marine and coastal scientists. Instead, they have to rely on the condensed data products and visualizations produced by the experts. These products and visualizations are often produced by various experts, during various projects, over many years. This makes it difficult to keep track of all the information that is available. And is, amongst others, hampering quality improvement and knowledge sharing as outlined in Nature [Merali, 2010]. In an ideal world, scientists and end-users have easy access to all raw data (from various data sources) as well as all derived products (e.g. Coastal State Indicators). Why is it not possible for end users to 'fly' through all data and CSIs at once, comparing the development of different indicators, for different time-periods and for different areas? Could this not support the decision making by managers and the draw up of expert advise by scientists and engineers? Matching this gap between specialist knowledge and end users has been discussed in literature [e.g. Van Koningsveld, 2003]. By developing and/or applying existing state-of-the-art techniques to share and visualize data we attempt to contribute to bridge this gap. In this paper we describe a first exploration to what extent all marine and coastal data and data products from NCK can be combined into one simple viewer for non-specialists. To do this we made use of the [OpenEarth] approach to data, models and tools. OpenEarth is the data management solution in [Building with Nature] and [MICORE]. We here present the result to the NCK-community to open a discussion with coastal scientists and managers regarding the additional value, future possibilities and future demands. What we are in fact looking for is an analog of YouTube for viewing marine and coastal data: 'DataTube'. The aim of this paper is to test whether such a 'DataTube' is already possible for a wide range of data, with existing technologies.

\section{Web Services for graphics: OGC WxS and KML}

At national, EU and international level many initiatives and projects are trying to enable non-specialists to visualize all kinds of geospatial data [Percivall, 2010]. The INSPIRE directive aims to provide a top-down overall framework for dissemination of all geospatial data in the EU [INSPIRE]. In the Netherlands a national registry was launched to host the meta-data of all 
governmental geospatial data, including marine and coastal data [NGR]. This website will link to 'DataTube' links were users can actually obtain the data. Generally, initiatives like these have chosen to rely on the international openGIS standards known as WxS. These standards have been issued by the [OGC] consortium to disseminate geospatial data over the web. WxS is the shorthand for a range of Web Services where the letter $\mathrm{x}$ denotes the specific standard. The most mature standard is WMS, short for Web Mapping Service [WMS]. WMS is meant for exchange of graphics of grid plots over the web. For the exchange of vector data, such as line elements and dots, there is a the WFS standard, short for Web Feature Service [WFS]. Separate WxS protocols exist for exchange of data over the web. Please refer to [Hankin et al, 2012; Baart et al., 2012] for a comparison of the three most mature ones: OGC's WCS, short for Web Coverage Service, OPeNDAP from the ocean community and GTS from the World Meteorological Organisation (WMO). However, our purpose here is only to enable plotting of marine and coastal data over the web, not exchange of the actual data. For our purpose WMS and WFS are sufficient. These two WxS protocols provide very powerful tools to visualize any data into viewers for the general public over the web. However, the specialists in the realm of marine and coastal science and engineering have hardly any experience with these protocols. Moreover, the actual implementation of these protocols in plotting (server) and viewing (client) software is lagging behind. Especially time-dependency, 3D views and nonorthogonal grids lag implementation. For instance, to our knowledge, time dependent WxS-complaint software for grids has only been implemented in pre-release software [Adaguc]. And curvi-linear and unstructured grids, very often used in marine and coastal models, are not supported by any existing WxS software yet. This means that these protocols are not appropriate yet for our aim, although their specifications were designed for this very purpose. Fortunately, another protocol is already capable of dealing with these aspects: [KML]. This is an open standard based on xml file format syntax and is recognized as standard by the

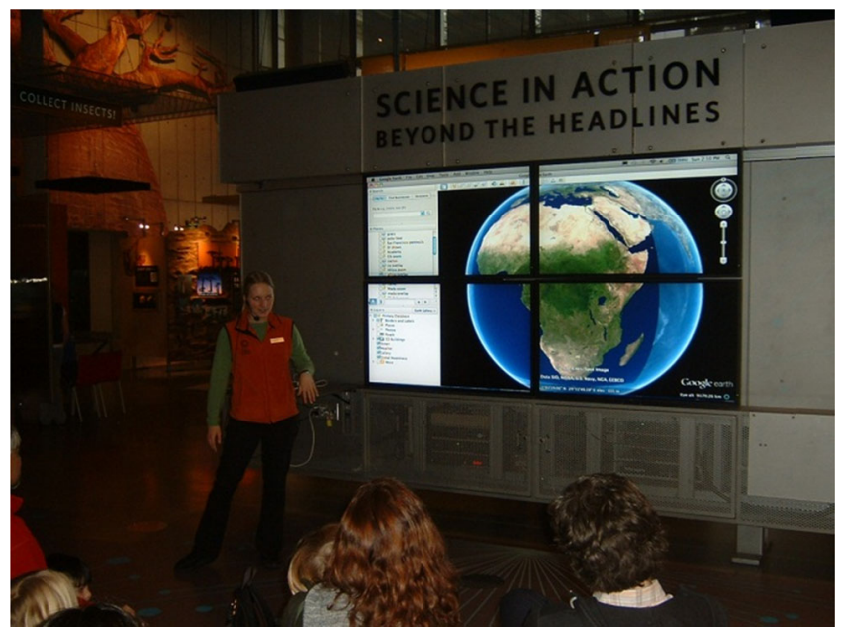

Fig. 1. A powerful example of DataTube-like outreach to general public: Google Earth show with a range of custom data for children in the brand-new California Academy of Sciences building, one of the largest museums of natural history in the world. Here a smooth map of Africa is overlaid on the Google Earth background. same OGC body that wrote the WxS services. However, KML is not in the repertoire of the top-down organizations that implement INSPIRE directive. KML, however, is the backbone of the simplest and most powerful viewer for the general public we could find: the free [Google Earth] viewer. In contrast to WMS and WFS viewers, this KML viewer is already well-known by endusers and the general public (Fig. 1)). (Google earth also supports stationary WMS.) KML is an open standard, so anyone can make a viewer that can interpret KML plots. Google Earth is the most advanced viewer available though. Currently, plotting data in Google Earth and encoding data as KML plots are therefore de facto the same. Note that because KML is an open standard, choosing KML does not necessarily mean limiting oneself to Google Earth. The Google Earth viewer has already implemented all aspects that are lagging for $\mathrm{WxS}$ viewers: $3 \mathrm{D}$, time-dependency and unstructured grids are already included. The aim of this paper is to test whether a 'DataTube' for all marine and coastal data is already possible with existing technologies. In this paper we chose to test whether the existing KML plotting standard with the Google Earth viewer can enable a DataTube for marine and coastal data.

\section{Reading guide}

In section 2 we introduce the community tools that we developed to plot data in KML (Google Earth). This software was written in Matlab, a commercial programming language that is used most widely by marine and coastal scientists and engineers in the NCK community. With the [OpenEarth] community [Van Koningsveld, 2010] we developed a generic, coherent toolbox for many atomic plot types in KML. In section 3 we test this toolbox with a wide range of large data sets currently used by the NCK community. We describe the specifics of the data and show whether we can plot it in KML in a manner that is readily useable by end-users. In section 4 we discuss the performance of plotting in KML, before we conclude in section 5 that plotting marine and coastal data in Google Earth can be considered a successful proof-of-concept solution to enable better collaboration between experts and endusers.

\section{METHOD: OET GOOGLEPLOT}

\section{OpenEarth community and workflow}

Matlab is one of the most commonly used programming languages for data analysis by marine and coastal scientist and engineers in NCK. In 2003, a number of scientists from Deltares and the TUDelft merged their Matlab toolboxes for marine and coastal science and engineering into one toolbox, culminating in open source release as OpenEarthTools (OET) in 2008. OpenEarth adopts the wikipedia approach to growth: web 2.0 crowd sourcing. All users are given full write access to help improve the collection. Quality is assured by version control tracking all changes in [SubVersion], the same tool used by professional software engineers worldwide. OpenEarth started as social experiment to investigate whether crowd sourcing was possible in our community. The answer is yes: over 1000 users registered, now enjoying over 5000 contributions from over 100 contributors. One of the most powerful toolboxes of OpenEarth is the GooglePlot toolbox. GooglePlot was developed in 307 revisions by 20 developers. They devised a set of Matlab functions that can plot 
any atomic data type with one coherent toolbox. The GooglePlot toolbox is designed to be the primary example of the OpenEarth quality control guidelines. For each GooglePlot plot function the settings were made customizable. Proper default values for all settings were chosen, which can be requested by calling the function without arguments. Common code fragments were detected in the toolbox and put into a separate subtoolbox. For each function in the GooglePlot toolbox a dedicated unit test was conceived. These test were coded into a unit test function that is run periodically to test ongoing performance of the entire toolbox. The rigorous test approach was included to deal with the typical shortcoming found in computer code made by scientists and engineers [Merali, 2010].

\section{Plot types in KML}

The first atomic data types to be implemented were the native plot types of Matlab. For intuitive use, the same nomenclature as Matlab was kept with KML as prefix: KMLline, KMLpcolor for 2D orthogonal and curvi-linear grids, KMLmesh/KMLsurf for 3D wire meshes and surfaces (flying carpets), KMLquiver for arrows, KMLmarker, KMLtext, KMLcontour derivatives for different contour lines, KMLtrimesh/KMLtrisurf for unstructured grids and KMLpatch for set of filled polygons. More atomic plot types were added in a later stage, going beyond the range offered by native Matlab plots: KMLcolumn/KMLcylinder, KMLcurtain for vertical time-space flying carpets, KMLcurvedarrrows for arrows that move in time and are curved following the streamlines. The latter function contains a dedicated particle-tracking implementation, with sophisticated seeding/decay algorithm. The most advanced function of GooglePlot is KMLfigure_tiler that can be used to chop up a grid datasets of any size into a collection of standard tiles [Thijs Damsma, pers.com.]. This method is known as a quad-tree [Wiliams, 1983] with universal texture, and is the very same technique that Google Earth uses view all the worlds satellite imagery. With this function, our toolbox has rivaled the capabilities of Google Earth imagery.

\section{Coordinate conversion}

Plotting in KML requires per definition latitude-longitude coordinates with the WGS84 datum, the same one used by GPS systems. KML and Google Earth have chosen to abandon support for the zillion coordinate systems in use. The choice of supporting only one single global coordinate system has greatly facilitated the ease of disseminating data via Google Earth. End-users, unknown to the difficulties and pitfalls of different coordinate systems, now have zero chance of running into coordinate conversion issues. Instead, coordinate issues have to by solved by scientists and engineers who make the KML files. Although coordinate conversion is not native knowledge of marine and coastal scientists and engineers, acquainting oneself with coordinate systems is a relatively simple task to them. Marine and coastal raw data is often only available in x-y coordinates. For just the NCK community these include for already: Rijksdriehoek for nearshore, UTM31-ED50 and UTM31-WGS84 for offshore, Lambert for Belgian waters, a German system in the Ems, latitude-longitude on WGS84, ED50 and ETRS89 globes, and many custom satellite projections, often polar stereographic. OpenEarth contains the ConvertCoordinates toolbox that can now convert almost any coordinate type to WGS84 lat-lon [Maarten van Ormondt, pers. com.]. Now coordinates can be mapped in batch mode directly from the data that scientist and engineers work with. ConvertCoordinates is subject to the same rigorous test procedure as GooglePlot, including the single free calibration coordinate provided by the [Kadaster]. With these two toolboxes a powerful and reliable toolbox was created for the marine and coastal specialists in NCK. It enables them to plot with little effort any kind of marine and coastal data in KML.

\section{RESULTS: A RANGE OF DATA}

\section{Does it work for the range of data sets in NCK?}

To test the OpenEarth GooglePlot toolbox, we choose a wide range of large datasets that that covers the usage and availability for marine and coastal scientists and engineers in the Dutch NCK community. We selected freely available datasets that underpin the research and advice of almost everyone in the NCK community. These are key assets of NCK in our opinion. And these are the sets are worth disseminating to decision makers and the general public. For each of these datasets an attempt is made to plot it as KML into Google Earth. The kmls are publicly available via $\mathrm{http}: / / \mathrm{kml}$.deltares.nl. In section 4 we will analyze to what extent we succeeded.

\section{Maps: North Sea grain sizes (Geological Survey of NL)}

The Geological Survey of the Netherlands (TNO/Deltares) compiled grain size maps of the bed of the Dutch part of the North Sea [Sytze van Heteren, pers. com]. These maps have a resolution of 200m and are available for D10, D50 and D90 grain sizes and $\%$-occurrence maps for mud and cobbles. These maps were compiled from a dataset of over 13,000 sediment cores/grabs [DINO]. This test set represents all time-independent map products such as DEMs and GIS rasters. It is shown to work successfully in KML in Fig. 2.

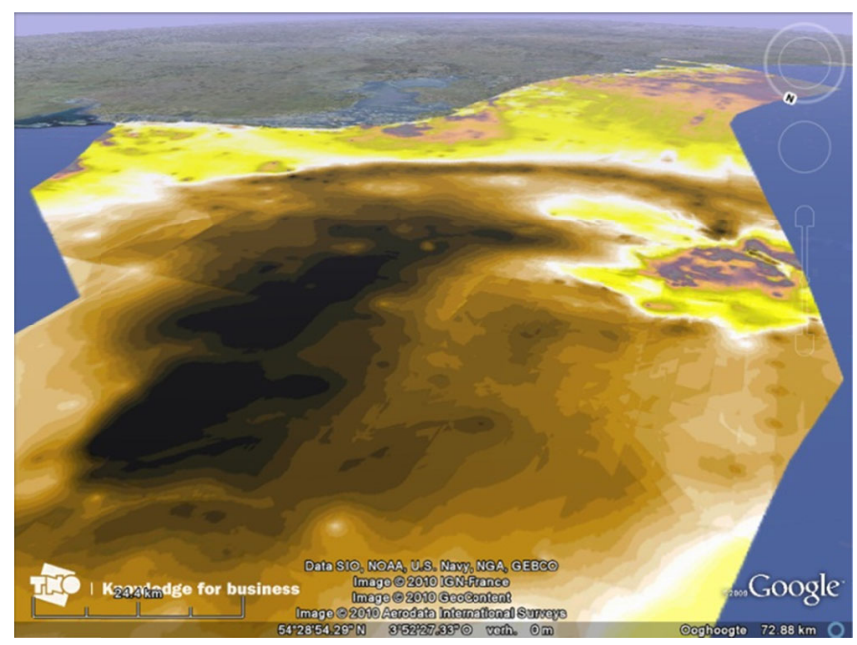

Fig. 2. D50 grain sizes, seen from central North Sea towards Europe. Data credit: Geological Survey of the Netherlands (TNO/Deltares). Image credit: Google Earth ${ }^{T M}$ mapping services.

\section{Transect time stacks: JarKus (Rijkswaterstaat)}

Since 1965 Rijkswaterstaat annually collects cross shore dune profiles along the entire Dutch coast at $100 \mathrm{~m}$ alongshore intervals [Minneboo, 1995]. The data covers the area inland behind the first dune row to an offshore water depth over $8 \mathrm{~m}$, with $5 \mathrm{~m}$ 
resolution. The data product is currently generated from shipbased soundings offshore, a dedicated device in the swash zone and Lidar in the dry foreshore and the dunes. The near shore sand bar zone is annually updated, whereas the datasets extends to a depth $8 \mathrm{~m}$ only at 5 or 10 year intervals only (doorlodingen). This test set represents all kinds of profile data and is shown to work successfully in KML in Fig. 3.

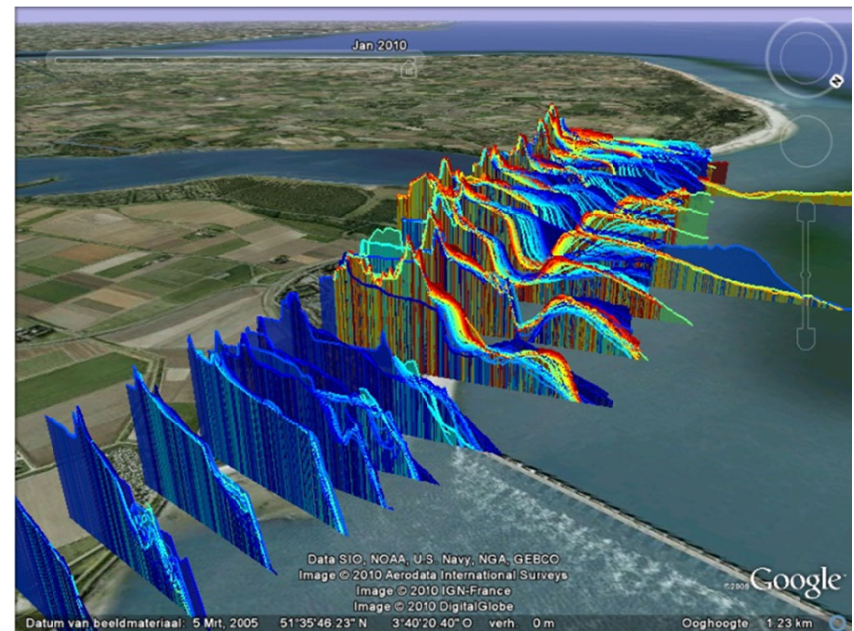

Fig. 3. Plot of JarKus profiles around the Eastern Scheldt barrier from 1965 to 2010 with a cross-shore resolution of $5 \mathrm{~m}$. The color represents the time at which a profile was collected (blue =old, red=recent). Data credit: Rijkswaterstaat. Plot credit: Claire van Oeveren Theeuwes (Deltares). Image credit: Google Earth ${ }^{T M}$ mapping services. Note the time slider.

\section{Map time stacks: vaklodingen bathymetry (Rijkswaterstaat)}

Since 1927 Rijkswaterstaat makes bathymetry soundings of the Dutch waters from deeper water up to the beach and into the tidal inlets of Zeeland and the Wadden Sea [Wiegmann et al., 2005]. Data is available as orthogonal grids with $20 \mathrm{~m}$ resolution at time intervals of about 7 years. This time dependency is needed due to the large morphological changes in the sedimentary beds of the Dutch waters. The early data consists of digitized navigation charts originally compiled from lead lines, the most recent are based on single and multi beam surveys. The product is chopped up into more almost 180 tiles known as "kaartblad", which are offshore extrapolations of the standard $10 \mathrm{~km} \times 12 \mathrm{~km}$ tiles from the 1:25.000 Dutch Topographic map definitions [Kadaster]. This bathymetry data is used to make legally binding sand balances; an important analyses in the context of the Dutch sustainable coastal policy (maintaining the sediment buffer in the so-called coastal foundation). The vaklodingen are shown to work successfully in KML in Fig. 4. To highlight small vertical variations a 500 element long colormap was developed with shading effects on top [Thijs Damsma, pers.com.]. A second time-dependent grid set is shown to work successfully in KML in Fig. 5: The Kusthoogte dataset with a resolution of $5 \mathrm{~m}$. This dataset is also available as tiles, but smaller ones: $5 \mathrm{~km}$ by $6 \mathrm{~km}$ tiles from the 1:10.000 Dutch Topographic map definitions. Both datasets are very large. The raw ASCII vaklodingen data are about $23 \mathrm{~GB}$, in netCDF it reduces to ca. $4 \mathrm{~GB}$. The raw Kusthoogte data is $2 \mathrm{~GB}$, because they are already stored in binary ArcGIS files. Viewing these complete datasets for all times at once would require GBs of memory on the computer where Google Earth runs. However, $4 \mathrm{~Gb}$ is beyond the physical limit of 32 bit PCs. Only the native Google Earth quad-tree with universal texture implemented in the GooglePlot KMLfigure_tiler function allows end-users to view these data in Google Earth. For 2D data plotted in this type, Google Earth only loads the subset that is actually visible. 3D plotting of all data within view is again beyond the capacities of normal PCs. We chose a successful approach with a light KML overview of the boundary boxes of all tiles (Fig. 6) with links to 3D KML visualizations of individual tiles at one time (Fig. 7).

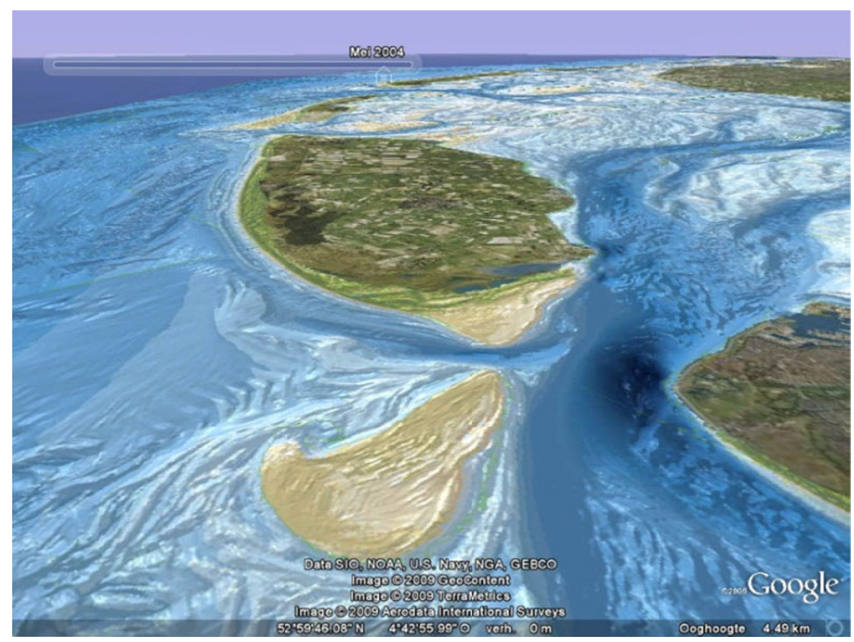

Fig. 4. Map of bathymetry in Western Wadden Sea in May 2004. Data credit: Rijkswaterstaat. Plot credit: Thijs Damsma (Van Oord). Image credit: Google Earth ${ }^{T M}$ mapping services. Note the time slider.

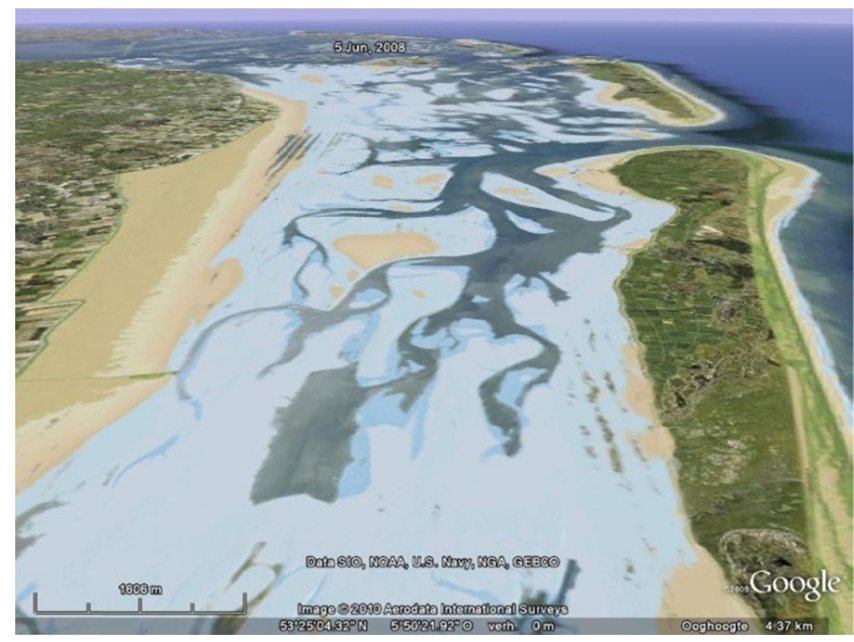

Fig. 5. Map of Kusthoogte bathymetry dataset in 2008. This dataset was collected with Lidar at a very low water and binned to cells of $5 \mathrm{~m}$. Lidar can only get bathymetry soundings for locations that are dry, therefore there are data gaps in the channels. Data credit: Rijkswaterstaat. Image credit: Google Earth $^{T M}$ mapping services. Note the time slider. 


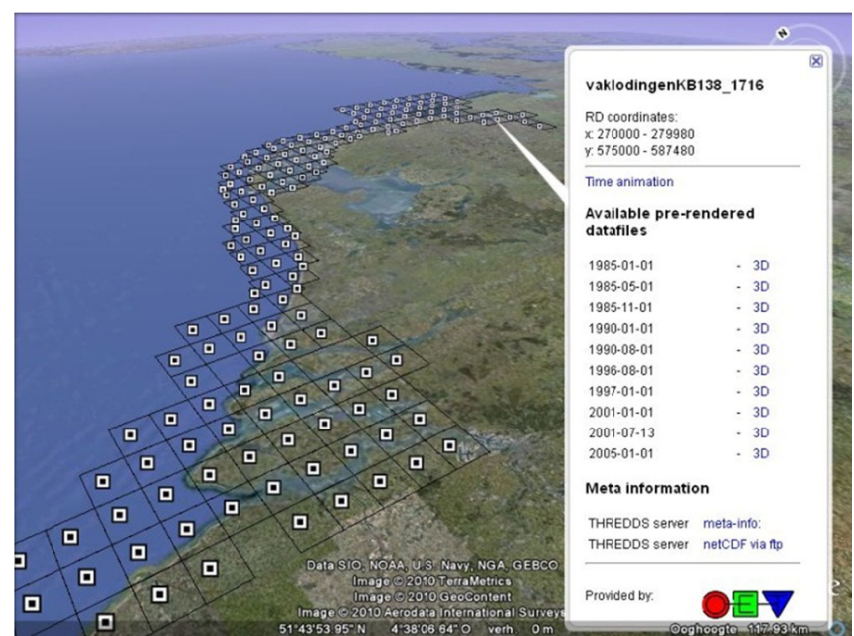

Fig. 6. Overview map of vaklodingen with spatio-temporal metadata and links to $3 D$ data of individual tiles. Data credit: Rijkswaterstaat. Image credit: Google Earth ${ }^{T M}$ mapping services.

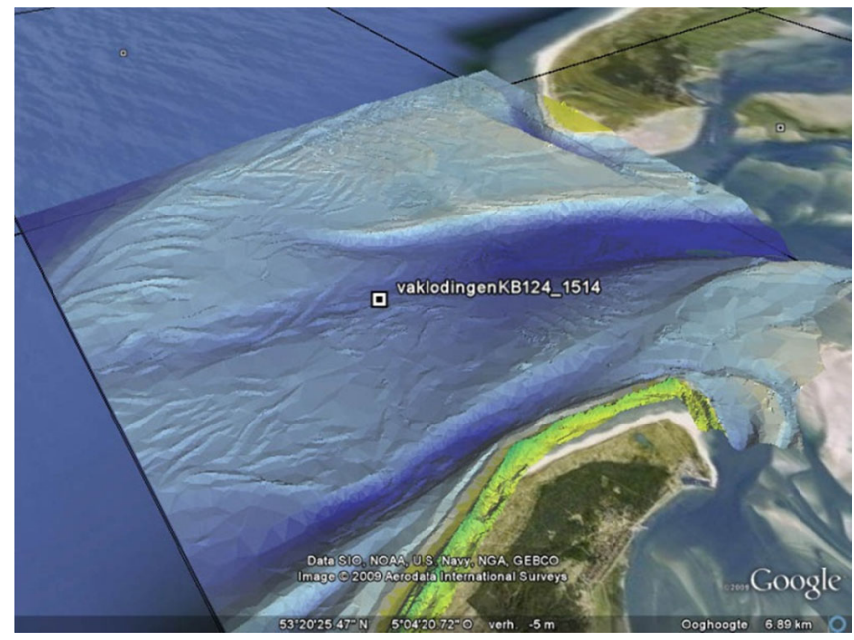

Fig. 7. $3 D$ visualization that allows for a 'fly through' of one vaklodingen tile at one time instance. Data credit: Rijkswaterstaat. Image credit: Google Earth ${ }^{T M}$ mapping services. Plot credit: Thijs Damsma (Van Oord).

\section{Time series (Rijkswaterstaat)}

Since the late 1900's Rijkswaterstaat records water levels offshore, along the coast and at inland waters. Initially only low and high water were recorded, but modern data are available at 10 min. intervals. For some stations over 100 years continuous recordings is available. Since the 60 's, water quality parameters are collected at the water surface on a weekly basis, reduced to bimonthly at some locations now due to gradually improved water quality in Europe. This set is called MWTL (Monitoring Waterstaatkundige Toestand des Lands). Recorded parameters include salinity, temperature, suspended sediment concentrations, $\mathrm{pH}$, Chlorophyll, and many nutrients and pollutants. Since the 80 's also wave periods, directions and heights are available from about 6 buoys. Gradually time series in the central North Sea become available for physical parameters via international collaboration and oil companies. The time series are stored in the offline DONAR database from Rijkswaterstaat, and available via a dedicated web service [Waterbase]. We show all locations of the
MWTL water quality stations to work successfully in KML in Fig. 8. For each dot a meta-data pop-up appears as shown in Fig. 9.

\section{Lines: Dutch beach lines (Rijkswaterstaat)}

From the aforementioned JarKus dataset dedicated Coastal State Indicators (CSI) are extracted. A CSI typically yields one number per cross shore transect. Since 1990, the Dutch coastal policy aims at preventing further retreat of the coastline. Therefore, the yearly Momentary Coastline (MKL) and the Testing Coastline (TKL) are derived from the Jarkus dataset. In case of structural exceedance of the reference coastline (BasisKustlijn BKL), local nourishments are considered. In addition, the position of low water, high water and dune foot and many other indicators [Santinelli et al., this NCK volume] are derived from JarKus. The CSIs can simply be plotted as time dependent alongshore lines, i.e. shape files. In Fig. 10 they are shown to work successfully in KML.

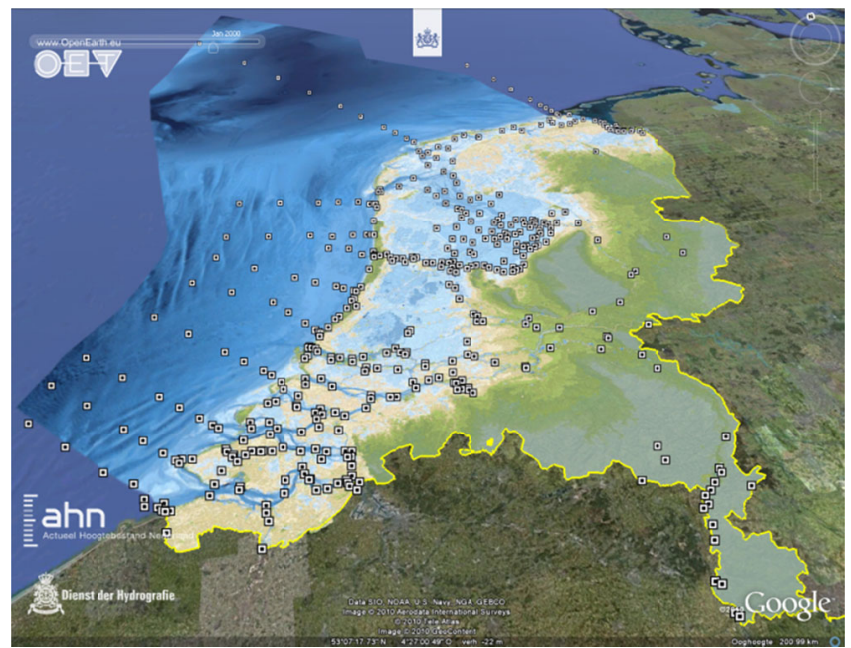

Fig. 8. Locations of MWTL water quality measuring locations on top of the $200 m$ North Sea bathymetry from the Hydrographic Office (www.hydro.nl) and the $100 \mathrm{~m}$ MaaiVeld set from TNO. Data credit: Rijkswaterstaat. Image credit: Google Earth ${ }^{T M}$ mapping services.
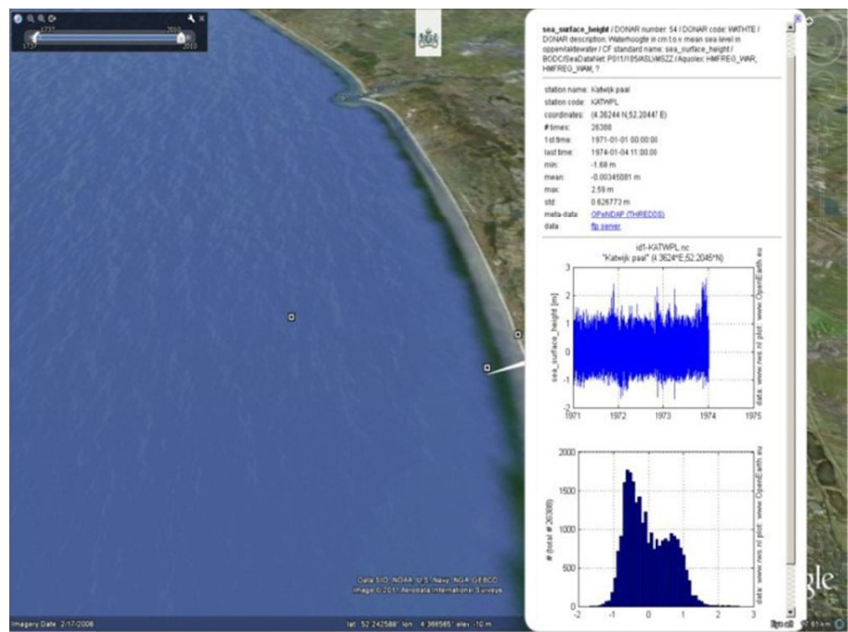

Fig. 9. For each location in Fig. 6 a pop-up is available with statistical data and a preview of the time series and a histogram. Data credit: Rijkswaterstaat. Image credit: Google Earth ${ }^{T M}$ mapping services. 


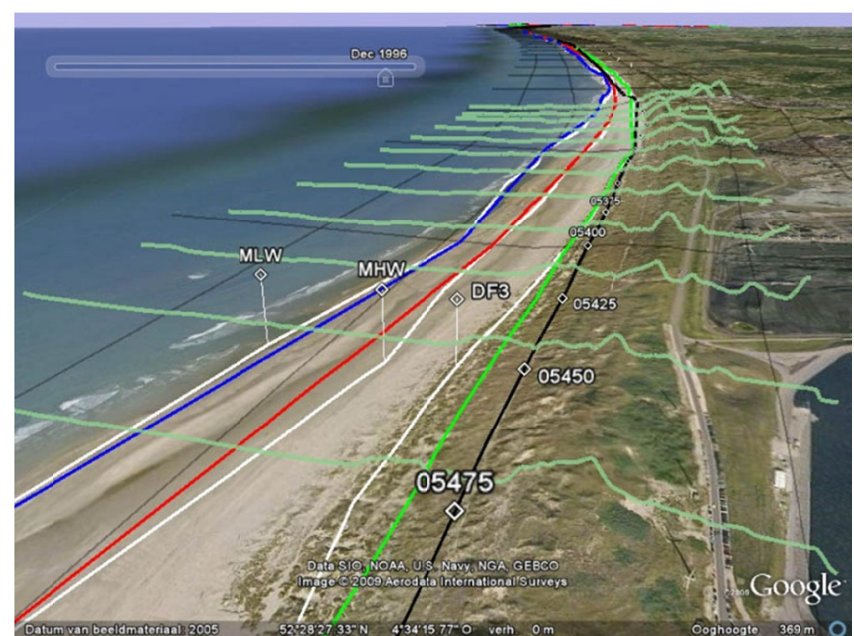

Fig. 10. Example of JarKus profiles in one year (viz. Fig. 3) with beach line indicators overlaid. Data credit: Rijkswaterstaat. (DF3 $=$ Dune Foot $3 \mathrm{~m})$. The alongshore numbers on the black line indicate the beach poles (strandpaal). Data credit: Rijkswaterstaat + Deltares. Image credit: Google Earth ${ }^{T M}$ mapping services.

Scatter points: Sieve curves Wadden Sea (Rijkswaterstaat)

Nearly 9000 sediment cores (surface grabs) were collected in the Wadden Sea by Rijkswaterstaat in the 80's and 90's. These data are part of the Sediment Atlas Wadden Sea executable that is available for download [sediment atlas] and allows for data export as txt file. This set is included to test performance of OpenEarth for any kind of data with a non-spatial or temporal dimension, such as grain sizes/classes, wave directions, wave frequencies. In Fig. 9 they are shown to work successfully in KML.

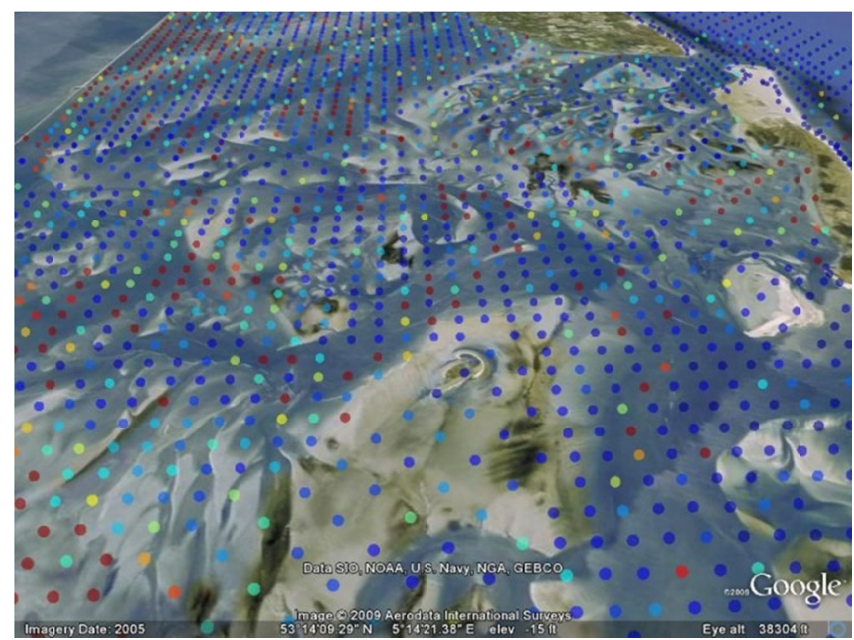

Fig. 11. The mud percentage in the bed in the Western Wadden Sea, calculated from data in the Sediment Atlas. Data credit: Rijkswaterstaat. Image credit: Google Earth ${ }^{T M}$ mapping services.

\section{North Sea sediment samples (Geological Survey of NL)}

The North sea grain size maps of the North Sea were compiled using sediment cores and surface grabs of the North Sea bed. These datasets contain the primary sediment type per sample layer. In Fig. 12 they are shown to work successfully in KML. For these data a dedicated OET GooglePlot function was developed to be able to plot these cores as 3D cylinders.

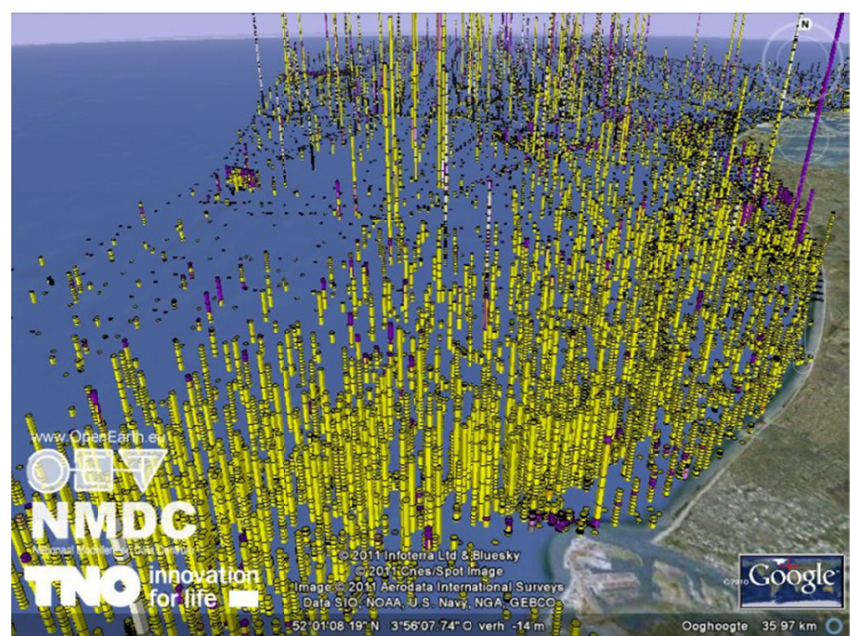

Fig. 12. All $>13,000$ open sediment cores data available on the North Sea plotted simultaneously. Data credit: Geological Survey of the Netherlands [DINO] via [NODC] format. Plot credit: Gerben de Boer (Deltares in NMDC context). Image credit: Google Earth ${ }^{T M}$ mapping services.

\section{Nourishments (Rijkswaterstaat)}

The Dutch coast has been eroding; over more than a thousand years. Coastal retreat puts coastal functions (e.g. safety against flooding) under pressure. Since 1990, the Dutch policy aims at structural prevention of a further retreat of the coastline, but in the meantime taking the valuable dynamical behavior of the coast into account. Therefore, sand nourishments have been preferred over hard structures to counteract the systematic erosion. Since 1952 Rijkswaterstaat registered the nourishments along the Dutch coast. To analyze the morphological development of the Dutch coast, this nourishment record is essential (as large amounts of sediments have been nourished). Therefore the nourishment database is an essential dataset for NCK research. In Fig. 13 it is shown to work successfully in KML.

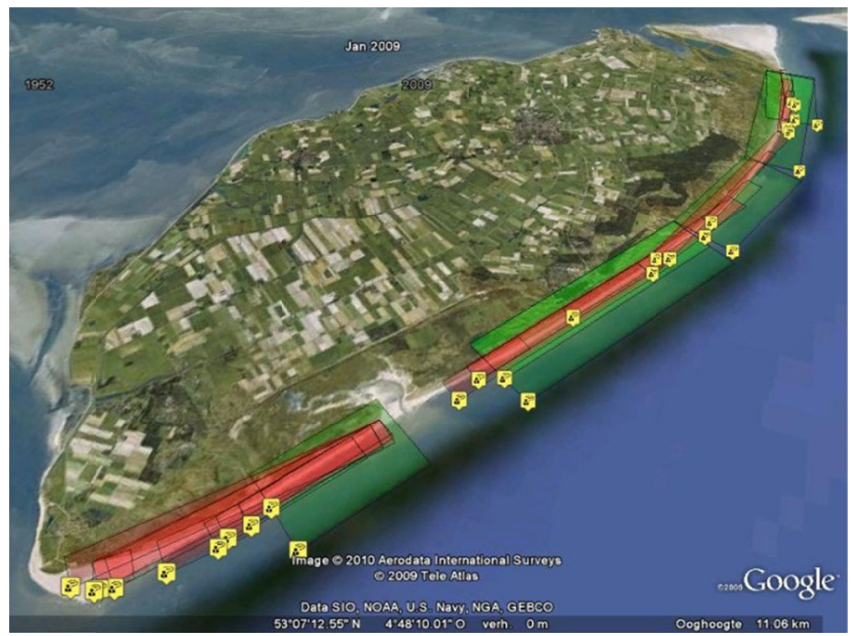

Fig. 13. Beach and foreshore suppletions since 1952. The area indicates the sand volume (assumed $1 \mathrm{~m}$ thick), the color indicates the nourishment type (red=beach, green=under water). Data credit: Rijkswaterstaat. Image credit: Google Earth ${ }^{T M}$ mapping services. 


\section{CONCLUSION: DATATUBE WORKS}

The aim of this paper was to test whether it is possible to plot a wide range of data from the NCK community for easy viewing by end-users and the general public. The successful examples in section 3 have shown that the existing technologies, KML in combination with the OpenEarth community toolboxes, are capable of plotting almost any data type for ready viewing by nonspecialist users. The opportunities for outreach by NCK are abundant. We can consider a 'DateTube' feasible.

\section{DISCUSSION: DATA DELUGE SEARCH}

We started this paper by stating that there is a gap between specialist knowledge and end-users. The difficulty to keep track of all information from experts was the main cause for this. Plotting of all data in KML has solved this issue. However, the gap between specialists and end-users does not appear to have ceased. In contrary, feed-back from end-users learned us that a new challenge has arisen. The supply of readily available data in Google Earth is now so huge, that end-users have difficulty in finding the correct data. They are basically overwhelmed and have expressed a need for filters to group data into manageable clusters for specific purposes. Our experiment has shown that giving away data has created more work for experts: only they have the knowledge to do this filtering. Some experts are still reluctant to open up their data though. They fear that their expert work might not be needed any more. Our pilot DataTube has showed the opposite. Anyone opening up his data will immediately be contacted by the overwhelmed end-users. In the past, control over data access was profitable, in the future only control of data filters will become valuable. The next challenge for marine and coastal scientists and engineers is therefore to create search options for end-users to find the correct KMLs. Top-down INSPIRE-like philosophies propose to make central inventories, catalogs, that allow to search data. There is a special OGC protocol for this: CSW: Catalogue Service for Web. However, we think it will become a challenge to keep such central catalogues up to date with the speed at which end-users can now generate new KML files. We believe that a different search option is worth investigating too: regular search enginers. We have shown that viewing data is now as simple as viewing YouTube. YouTube offers a similar deluge of information as a collection of KML files, and it is not subject to complaints from overwhelmed users. The reason is that YouTube offers a simple and powerful search box to search the movies. We therefore envision a future where KML should be as easy as to search as movies. Our next challenge to therefore to tag each KML with specific micro-information and user votes, so that general search engines can find them as easy as YouTube movies. Only then DataTube is complete: open data and simple searches.

\section{REFERENCES}

Adaguc, http://adaguc.knmi.nl.

AHN, Actueel Hoogtebestand Nederland, http://www.ahn.nl.

Baart, F.; de Boer, G.J.; de Haas, W.; Donchyts, G.; Philippart, M.; van Koningsveld, M.; Plieger, M., 2012. A comparison between WCS and OPeNDAP for making model results and data products available through the internet. Trans. in GIS, accepted.

Building with Nature, http://www.ecoshape.nl.
DINO, http://www.dinoloket.nl.

e-IRG, 2009. Report on data management, e-IRG Data management Task Force, http://www.e-irg.eu.

Google Earth, http://earth.google.com.

Hankin S.C., Blower J.D., Carval Th., Casey K.S., Donlon C., Lauret O., Loubrieu Th., Srinivasan A., Trinanes J., Godoy O, Mendelssohn R., Signell R., De La Beaujardiere J., Cornillon P., Blanc F., Rew R., Harlan J. (2010). NETCDF-CFOPENDAP: standards for ocean data interoperability and object lessons for community data standards processes. Oceanobs 2009, Venice Convention Centre, 21-25 Sep. 2009, Venice. http://archimer.ifremer.fr/doc/00027/13832.

INSPIRE directive, http://inspire.jrc.ec.europa.eu.

Kadaster, kernnetpunt, https://rdinfo.kadaster.nl/pics/publijst1.gif.

KML, http://www.opengeospatial.org/standards/kml.

Merali, Z, 2010. Error... Why scientific computing does not compute. Nature, 467 775-777.

MICORE, http://www.micore.eu.

Minneboo, F.A.J., 1995. Jaarlijkse kustmetingen Rijkswaterstaat RIKZ, rapport RIKZ-95.022.

NGR, Nationaal GeoRegister, http://www.nationaalgeoregister.nl.

NODC, http://www.nodc.nl..

OGC, http://www.opengeospatial.org.

OpenEarth, http://www.OpenEarth.nl.

Percivall, G., 2010. The application of open standards to enhance the interoperability of geoscience information. International Journal of Digital Earth, 3(1), 14-30.

Sediment Atlas Wadden Zee, http://www.waddenzee.nl.

SubVersion, http://subversion.tigris.org/.

Van Koningsveld, M., de Boer, G.J., Baart, F., Damsma, T., den Heijer, C., van Geer, P., de Sonneville, B., 2010. OpenEarth inter-company management of: data, models, tools \& knowledge.WODCON XIX, September 2010. China, Beijing.

Van Koningsveld, M., 2003. Matching specialist knowledge with end user needs. Bridging the gap between coastal science and coastal management. $\mathrm{PhD}$ thesis, Twente University, Enschede, The Netherlands. ISBN 90-365-1897-0.

Waterbase, Rijkswaterstaat, http://live.waterbase.nl. WFS, http://www.opengeospatial.org/standards/wfs.

Wiegmann, N. R. Perluka, S. Oude Elberink, J. Vogelzang, 2005. Vaklodingen: de inwintechnieken en hun combinaties. AGI Rijkswaterstaat, Rapp.Nr. AGI-2005-GSMH-012.

Wiliams, 1983, Pyramidal parametrics, ACM Siggraph Computer Graphics, 1983, 17(3), 1-11.

WMS, http://www.opengeospatial.org/standards/wms.

\section{ACKNOWLEGDEMENTS}

Part of this study have been carried out (i) by Deltares in the framework of the KPP-B\&O Kust and KPP-Kustbeleid projects, both commissioned by Rijkswaterstaat Waterdienst, (ii) by Van Oord and Deltares in the data management case (DM) of Building with Nature, (iii) by TU Delft in the data management workpackage of the EU FP7 MICORE project, (iv) by Deltares in the Verbetertraject voor visualisatie en user interfaces of NMDC. Numerous other projects contributed parts as well. We are very grateful to the open marine and coastal data from Rijkswaterstaat and the Geological Survey of the Netherlands (TNO/Deltares). 